\title{
잔 Measurable Residual Disease Response and Prognosis in Treatment-Naïve Acute Myeloid Leukemia With Venetoclax and Azacitidine
}

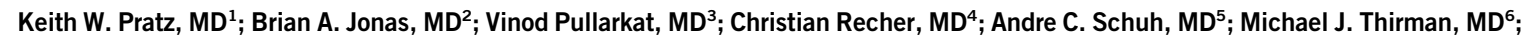 \\ Jacqueline S. Garcia, MD; Courtney D. DiNardo, MD ${ }^{8}$; Vladimir Vorobyev, MD ${ }^{9}$; Nicola S. Fracchiolla, MD ${ }^{10}$; Su-Peng Yeh, MD ${ }^{11}$; \\ Jun Ho Jang, MD ${ }^{12}$; Muhit Ozcan, MD ${ }^{13}$; Kazuhito Yamamoto, MD ${ }^{14}$; Arpad Illes, MD ${ }^{15}$; Ying Zhou, PhD ${ }^{16}$; Monique Dail, PhD ${ }^{17}$; \\ Brenda Chyla, PhD ${ }^{16}$; Jalaja Potluri, MD ${ }^{16}$; and Hartmut Döhner, MD ${ }^{18}$
}

PURPOSE There is limited evidence on the clinical utility of monitoring measurable residual disease (MRD) in patients with acute myeloid leukemia treated with lower-intensity therapy. Herein, we explored the outcomes of patients treated with venetoclax and azacitidine who achieved composite complete remission (CRc; complete remission + complete remission with incomplete hematologic recovery) and MRD $<10^{-3}$ in the VIALE-A trial.

METHODS The patients included in this report were treated with venetoclax and azacitidine. Bone marrow aspirate samples for multiparametric flow cytometry assessments were collected for central analysis at baseline, end of cycle 1 , and every three cycles thereafter. MRD-negative response was defined as $<1$ residual blast per 1,000 leukocytes ( $<10^{-3}$ or $0.1 \%$ ) with an estimated analytic sensitivity of $0.0037 \%-0.0027 \%$. CRc, duration of remission (DoR), event-free survival (EFS), and overall survival (OS) were assessed. A multivariate Cox regression analysis identified prognostic factors associated with OS.

RESULTS One hundred sixty-four of one hundred ninety (86\%) patients with CRc were evaluable for MRD. MRD $<10^{-3}$ was achieved by 67 of 164 (41\%), and 97 of 164 (59\%) had MRD $\geq 10^{-3}$. The median DoR, EFS, and OS were not reached in patients with $\mathrm{CRc}$ and $\mathrm{MRD}<10^{-3}$, and the 12-month estimates for DoR, EFS, and OS in this group were $81.2 \%, 83.2 \%$, and $94.0 \%$. Among patients with CRc and MRD $\geq 10^{-3}$, the median DoR, EFS, and OS were 9.7, 10.6, and 18.7 months. Multivariate analysis showed that CRc with MRD $<10^{-3}$ was a strong predictor of OS (adjusted hazard ratio $=0.285 ; 95 \% \mathrm{Cl}, 0.159$ to $0.510 ; P<.001$ ).

CONCLUSION Patients who achieved CRc and MRD $<10^{-3}$ with venetoclax and azacitidine had longer DoR, EFS, and $\mathrm{OS}$, than responding patients with MRD $\geq 10^{-3}$.

J Clin Oncol 00. ๑ 2021 by American Society of Clinical Oncology

Creative Commons Attribution Non-Commercial No Derivatives 4.0 License (c) (): $\Theta$

\section{INTRODUCTION}

The phase III VIALE-A trial established the treatment of venetoclax and azacitidine combination as a new standard of care for older patients with treatment-naïve acute myeloid leukemia (AML) who were ineligible for intensive chemotherapy. ${ }^{1,2}$ Results from the trial demonstrated that the combination of venetoclax and azacitidine led to a significant improvement in overall survival (OS) and composite complete remission (CRc; complete remission $[\mathrm{CR}]+$ complete remission with incomplete hematologic recovery [CRi]). The rates of CRc with measurable residual disease (MRD) response $\left(<10^{-3}\right)$ were also significantly higher in the venetoclax and azacitidine group as compared with azacitidine alone $(23.4 \% \vee 7.6 \%, P<0.001)$, providing evidence that deep responses are attainable in patients treated with lower-intensity therapy. ${ }^{2}$

Despite the achievement of morphological remission in most older patients with AML receiving standard intensive therapy, approximately $80 \%$ experience relapse because of residual leukemic cells in the bone marrow (BM). ${ }^{3-6}$ In intensively treated patients, the achievement of a deeper MRD-negative response in $\mathrm{CR}$ is a prognostic marker of superior outcomes, including OS and relapse-free survival, compared with conventional remission criteria alone $(<5 \%$ blasts by morphology). ${ }^{7,8} \mathrm{~A}$ retrospective study reported that in patients age $>60$ years and treated with hypomethylating agents, the cumulative incidence of relapse was lower among patients who had achieved a MRD-negative $\left(<10^{-3}\right)$ response, but failed to establish a relationship between MRD and OS or relapsefree survival. ${ }^{9}$ However, there is limited literature on the clinical significance of MRD, survival outcomes, and the utility of monitoring in treatment-naive patients receiving lower-intensity therapy when ineligible for intensive induction chemotherapy.

Immunophenotyping with multiparametric flow cytometry (MFC) is a powerful technique used to quantify MRD in AML. ${ }^{10,11}$ Although limitations of MFC-based 


\section{CONTEXT}

\section{Key Objective}

To evaluate the outcomes of patients who achieved composite complete remission (CRc, defined as complete remission and complete remission with incomplete hematologic recovery) and were evaluable for a measurable residual disease (MRD) response by multiparametric flow cytometry in patients with acute myeloid leukemia treated with venetoclax and azacitidine.

\section{Knowledge Generated}

The patients who achieved CRc + MRD-negative $\left(<10^{-3}\right)$ response with the combination had a longer duration of remission, overall survival (OS), and event-free survival. Achievement of $\mathrm{CRC}+\mathrm{MRD}<10^{-3}$ response was a prognostic factor for OS, providing evidence that MRD monitoring could be informative for patients who are treated with lowerintensity therapies.

\section{Relevance}

The outcomes of this analysis provide evidence that MRD assessments among patients treated with lower-intensity therapy could be conducted to understand the prognostic impact of MRD on OS. MRD $<10^{-3}$ response in patients treated with venetoclax and azacitidine is valuable and warrants further investigation to establish its role in clinical management.

MRD analyses include the lack of standardization, reliance on a high-quality marrow aspirate, and variable sensitivity, the method is applicable to a majority of patients after a treatment-induced morphologic remission, even for those who do not harbor an appropriate molecular target for quantitative polymerase chain reaction ${ }^{12}$ and nextgeneration sequencing (NGS) MRD approaches. ${ }^{13}$

In this report, we evaluated the outcomes of patients who achieved CRc and were evaluable for an MRD response by MFC in the aforementioned cohort of patients with AML treated with venetoclax and azacitidine in the VIALE-A trial. Among patients treated with placebo and azacitidine, only 11 of 145 patients achieved CRc with an MRD-negative $\left(<10^{-3}\right)$ response and thus were excluded from this exploratory analysis because of small numbers.

\section{METHODS}

\section{Patients and Treatment}

Patients enrolled in the ongoing randomized phase III VIALE-A study (NCT02993523) who received venetoclax and azacitidine (venetoclax arm) were included in this analysis. Enrolled patients were $\geq 18$ years with a confirmed diagnosis of AML by the WHO criteria and were ineligible for standard induction chemotherapy either because of age $\geq 75$ years or because of comorbidities. Additional eligibility criteria have been previously published. ${ }^{2}$ Patients in the venetoclax arm received venetoclax $400 \mathrm{mg}$ orally once daily on days 1-28 and azacitidine at $75 \mathrm{mg} / \mathrm{m}^{2}$ intravenously once daily on days $1-7$ once every 28-day cycle.

The study was approved by the local ethics committees and conducted in accordance with the International Conference on Harmonization, Good Clinical Practice guidelines, and the Declaration of Helsinki. All patients provided written informed consent.

\section{Outcomes}

Disease responses were evaluated per modified International Working Group response criteria for AML. ${ }^{14}$ Efficacy was assessed as CRc, duration of CRc, event-free survival (EFS), and OS. CR was defined as absolute neutrophil count $>10^{3} / \mu \mathrm{L}$, platelets $>105 / \mu \mathrm{L}$, red cell transfusion independence, and BM with $<5 \%$ blasts. CRi was defined as all criteria for CR, except for neutropenia $\leq 10^{3} / \mu \mathrm{L}$ or thrombocytopenia $\leq 105 / \mu \mathrm{L}$. Duration of remission (DoR) for $\mathrm{CRc}$ was defined as the number of days from the date of first response (CR or $\mathrm{CRi}$ ) per modified International Working Group criteria for AML to the earliest evidence of confirmed morphologic relapse, confirmed progressive disease, or death because of disease progression. EFS was defined as the number of days from random assignment to the date of confirmed progressive disease, confirmed morphological relapse from $\mathrm{CR}$ or $\mathrm{CRi}$, treatment failure (defined as a failure to achieve CR, CRi, or morphological leukemia-free state) after at least six cycles of study treatment, or death from any cause. OS was defined as the time from random assignment to the date of death from any cause. BM assessments were performed at screening, at end of cycle 1, and after every three cycles thereafter. Per protocol, for patients who had two successive disease assessments that indicated CR or CRi, BM aspirates were no longer required for response or biomarker assessments. The patients had to achieve an MRD-negative response before or after clinical remission, and the MRD response had to occur before study drug discontinuation. Adverse events were graded according to the National Cancer Institute Common Terminology Criteria for Adverse Events Version $4 \cdot 0 .{ }^{15}$

\section{Detection of MRD}

MRD response was defined as one or fewer residual leukemic blasts per 1,000 leukocytes or $10^{-3}$. Patients who 
had one negative sample for MRD value below this cutoff at any time on the study were defined as patients with an MRD-negative response. Samples were collected at baseline from BM aspirates during the clinical assessment and after each course as described above. Patients were unevaluable for MRD if they had missing BM samples, were deemed a technical failure, or if the BM samples contained less than a hundred thousand CD45+ leukocytes (classified thereafter as MRD-indeterminate). The analysis was performed centrally by LabCorp Central Laboratory Services and was blinded to investigator-reported disease response assessments. The BM aspirates were analyzed by a five-tube, eight-color panel (CD45, CD34, HLA-DR, and CD13 as a backbone in all five tubes, with CD123, CD117, CD71, CD64, CD56, CD38, CD33, CD19, CD15, CD14, $\mathrm{CD} 11 \mathrm{~b}, \mathrm{CD} 7, \mathrm{CD} 4$, and $\mathrm{CD} 2$ assessed across the remaining tubes). These markers align with the recent recommendations of the European LeukemiaNet consensus document for flow cytometry-based MRD assessments in AML. ${ }^{11}$ The integrated leukemia-associated immunophenotypes and different than normal procedures were used. ${ }^{10,11}$ The assay validation established the analytical sensitivity of the MRD panel at the upper limit of $0.0037 \%$ and the lower limit of $0.0027 \%$. For specimens in which the identified aberrant phenotypes were quantified below $7 \%$, the data were reviewed and approved by a board-certified hematopathologist; a total of four hematopathologists reviewed MRD assessments for this trial.

\section{Statistical Analysis}

Demographics were summarized by descriptive statistics. DoR, EFS, and OS were estimated by the Kaplan-Meier methodology between the $\mathrm{CRc}+\mathrm{MRD}<10^{-3}$ and $\mathrm{CRC}+\mathrm{MRD} \geq 10^{-3}$ groups. Median and $95 \% \mathrm{Cls}$ were reported. For subgroup analyses, hazard ratios (HRs) and 95\% Cls were generated using an unstratified Cox proportional hazards model. To improve the precision or HR estimates of OS, a multivariate Cox regression analysis was performed using adjustments of key prognostic factors as determined in the analysis of VIALE-A, including age, AML type, and cytogenetic risk.

\section{RESULTS}

As of January 04, 2020, 286 patients were randomly assigned into the venetoclax arm, of which 190 achieved CRc (Fig 1). Among patients who achieved CRc, 164 were evaluable for MRD, 22 patients had a missing MRD assessment, and four patients had indeterminate MRD values (Data Supplement, online only). An MRD-negative response was achieved by 67 , and 97 had persistent MRD $\geq 10^{-3}$. The clinical and molecular characteristics of patients are summarized in Table 1. Among patients with molecular mutations, the $\mathrm{CRc}$ and $\mathrm{MRD}$-negative response rates were $50 \%$ (10 of 20) for patients with $F L T 3,49 \%$ (21 of 43 ) for patients with IDH1/2, 30\% (6 of 20) for patients with TP53, and $88 \%$ (15 of 17) for patients with an NPM1 mutation.

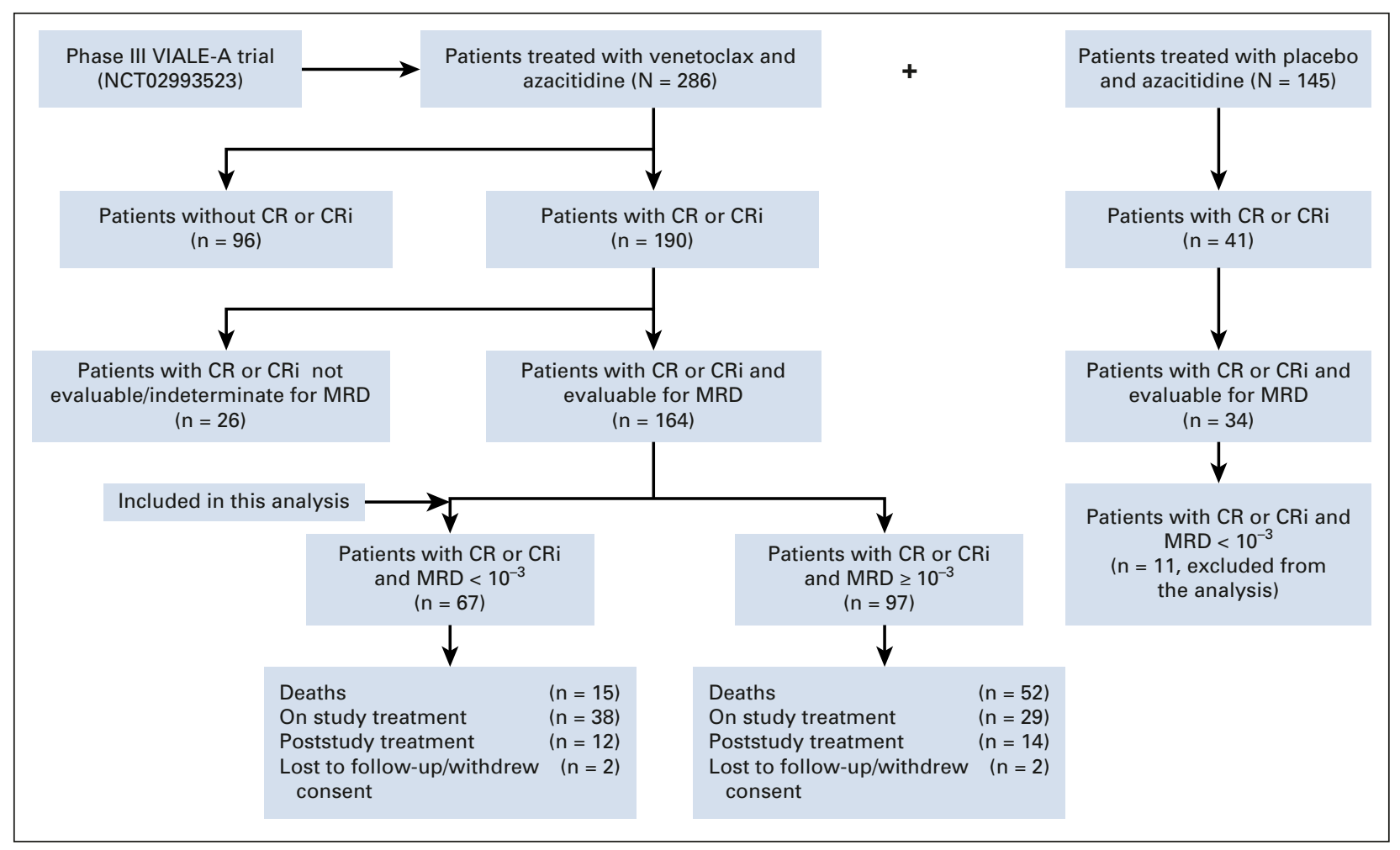

FIG 1. Profile of patients. CR, complete remission; CRi, complete remission with incomplete marrow recovery; MRD, measurable residual disease. 
TABLE 1. Baseline and Clinical Characteristics of Patients

CR + CRi and

MRD Evaluable $(n=164)$,

No. $(\%)$
CR + CRi and

MRD $<10^{-3}(n=67)$,

No. (\%)
CR + CRi and

$M R D \geq 10^{-3}(n=97)$,

No. (\%)
$P$ Value ${ }^{\mathrm{a}} \mathrm{CR}+\mathrm{CRi}$ and MRD $<10^{-3}$ ( $\mathrm{n}=67) v \mathrm{CR}+\mathrm{CRi}$ and MRD $\geq 10^{-3}$ $(\mathrm{n}=97)$

\begin{tabular}{|c|c|c|c|c|}
\hline \multicolumn{5}{|l|}{$\begin{array}{l}\text { Age categories (reported } \\
\text { from EDC), years }\end{array}$} \\
\hline 18 to $<65$ & $3(1.8)$ & $2(3.0)$ & $1(1.0)$ & .081 \\
\hline$\geq 75$ & $108(65.9)$ & $38(56.7)$ & $70(72.2)$ & \\
\hline \multicolumn{5}{|l|}{ Sex } \\
\hline Male & $95(57.9)$ & $34(50.7)$ & $61(62.9)$ & \\
\hline \multicolumn{5}{|l|}{ ECOG performance status } \\
\hline 0 & 31 (18.9) & $13(19.4)$ & $18(18.6)$ & .670 \\
\hline 1 & $70(42.7)$ & $25(37.3)$ & $45(46.4)$ & \\
\hline 2 & $57(34.8)$ & $26(38.8)$ & $31(32.0)$ & \\
\hline Poor & $48(29.3)$ & $16(23.9)$ & $32(33.0)$ & \\
\hline \multicolumn{5}{|l|}{ BM blast count, \% } \\
\hline$<30$ & $55(33.5)$ & $17(25.4)$ & $38(39.2)$ & .189 \\
\hline$\geq 30-<50$ & $33(20.1)$ & $15(22.4)$ & $18(18.6)$ & \\
\hline$\geq 50$ & $76(46.3)$ & $35(52.2)$ & $41(42.3)$ & \\
\hline \multicolumn{5}{|l|}{ Type of AML } \\
\hline De novo AML & $120(73.2)$ & $49(73.1)$ & $71(73.2)$ & 1.000 \\
\hline Secondary AML & $44(26.8)$ & $18(26.9)$ & $26(26.8)$ & \\
\hline ITD or TKD & $20(15.5)$ & $10(20.0)$ & $10(12.7)$ & .320 \\
\hline Not detected & $109(84.5)$ & $40(80.0)$ & 69 (87.3) & \\
\hline Undetermined or missing & 35 & 17 & 18 & \\
\hline \multicolumn{5}{|l|}{ IDH1 or IDH2 mutation ${ }^{b}$} \\
\hline IDHI & 12 (7.9) & $5(8.1)$ & $7(7.8)$ & \\
\hline $\mathrm{IDH} 2$ & $32(21.1)$ & $16(25.8)$ & $16(17.8)$ & \\
\hline IDH1 or IDH2 & $43(28.3)$ & $21(33.9)$ & $22(24.4)$ & \\
\hline Not detected & $109(71.7)$ & $41(66.1)$ & 68 (75.6) & .272 \\
\hline Undetermined or missing & 12 & 5 & 7 & \\
\hline \multicolumn{5}{|l|}{ TP53 mutation ${ }^{c}$} \\
\hline Detected & $20(19.6)$ & $6(14.3)$ & $14(23.3)$ & \\
\hline Not detected & $82(80.4)$ & $36(85.7)$ & $46(76.7)$ & \\
\hline Undetermined or missing & 62 & 25 & 37 & .316 \\
\hline
\end{tabular}

(continued on following page) 
TABLE 1. Baseline and Clinical Characteristics of Patients (continued)

\begin{tabular}{|c|c|c|c|c|}
\hline Characteristic & $\begin{array}{c}\text { CR + CRi and } \\
\text { MRD Evaluable }(n=164) \\
\text { No. }(\%)\end{array}$ & $\begin{array}{c}\text { CR + CRi and } \\
\text { MRD }<10^{-3}(n=67), \\
\text { No. }(\%)\end{array}$ & $\begin{array}{c}\text { CR + CRi and } \\
\text { MRD } \geq 10^{-3}(n=97), \\
\text { No. }(\%)\end{array}$ & $\begin{array}{c}P \text { Value }^{\mathrm{a}} \mathrm{CR}+\mathrm{CRi} \text { and MRD }<10^{-3} \\
(\mathrm{n}=67) v \mathrm{CR}+\mathrm{CRi} \text { and } \mathrm{MRD} \geq 10^{-3} \\
(\mathrm{n}=97)\end{array}$ \\
\hline \multicolumn{5}{|l|}{ NPM1 mutation ${ }^{c}$} \\
\hline Detected & $17(16.7)$ & $15(35.7)$ & $2(3.3)$ & $<.001$ \\
\hline Not detected & $85(83.3)$ & $27(64.3)$ & $58(96.7)$ & \\
\hline Undetermined or missing & 62 & 25 & 37 & \\
\hline
\end{tabular}

NOTE. Bold value indicates statistical significance.

Abbreviations: AML, acute myeloid leukemia; BM, bone marrow; CMML, chronic myelomonocytic leukemia; CR, complete remission; CRi, complete remission with incomplete marrow recovery; ECOG, Eastern Cooperative Oncology Group; MDS, myelodysplastic syndrome; MRD, measurable residual disease.

${ }^{a}$ The $P$ value provided is nominal as it was not included in the planned analysis. The Wilcoxon rank-sum test is used to compare continuous variables; Fisher's Exact test is used to compare categorical variables.

${ }^{\mathrm{b}} \mathrm{DH} 1$ or IDH2 and FLT3 data were determined by the CDx assay. ${ }^{2}$

${ }^{\mathrm{C}}$ TP53 and NPM1 data were from the central laboratory and determined by the MyAML assay. ${ }^{2}$

Overall, the baseline characteristics were similar among groups. However, the patients in the MRD-negative response group had a significantly higher rate of NPM1 mutation than did patients in the MRD $\geq 10^{-3}$ group (36\% $\vee 3 \%$ ).

Patients with $\mathrm{CRC}$ and evaluable for MRD had a median of 3.0 (range: 1.0-8.0) MRD assessments postbaseline. CRc and MRD-negative responses were achieved by 17 (25\%) patients by end of cycle 1, 18 (27\%) patients by end of cycle 4, 18 (27\%) patients by end of cycle 7, and 14 (21\%) patients after cycle 7 . The rate of CRc and MRD-negative response by treatment cycle and the cumulative incidence is depicted in Figure 2.

The median follow-up was 22.1 (range: 1.3-30.1) and 20.8 (range: 2.3-30.7) months in patients with MRD-negative response and $M R D \geq 10^{-3}$, respectively. Patients who attained an MRD-negative response at any time received a median treatment with 16.0 (range: 1.0-28.0) cycles of venetoclax and azacitidine, whereas patients with MRD $\geq 10^{-3}$ received a median treatment of 9.0 (range: 2.030.0) cycles.

The median DoR for $\mathrm{CRc}$ was not reached among patients with an MRD-negative response, and the 12-month estimated DoR for CRc and MRD-negative response was 81.2\% (95\% Cl, 69.3 to 88.9). The median DoR for CRC was $9.7(95 \% \mathrm{Cl}, 8.0$ to 15.8$)$ months in patients with MRD $\geq 10^{-3}$ (Fig 3A). Subgroup analysis by age ( $\geq 75 \mathrm{v}$ $<75$ years), AML subtype (de novo $v$ secondary), and cytogenetic risk (poor $v$ intermediate) showed that the

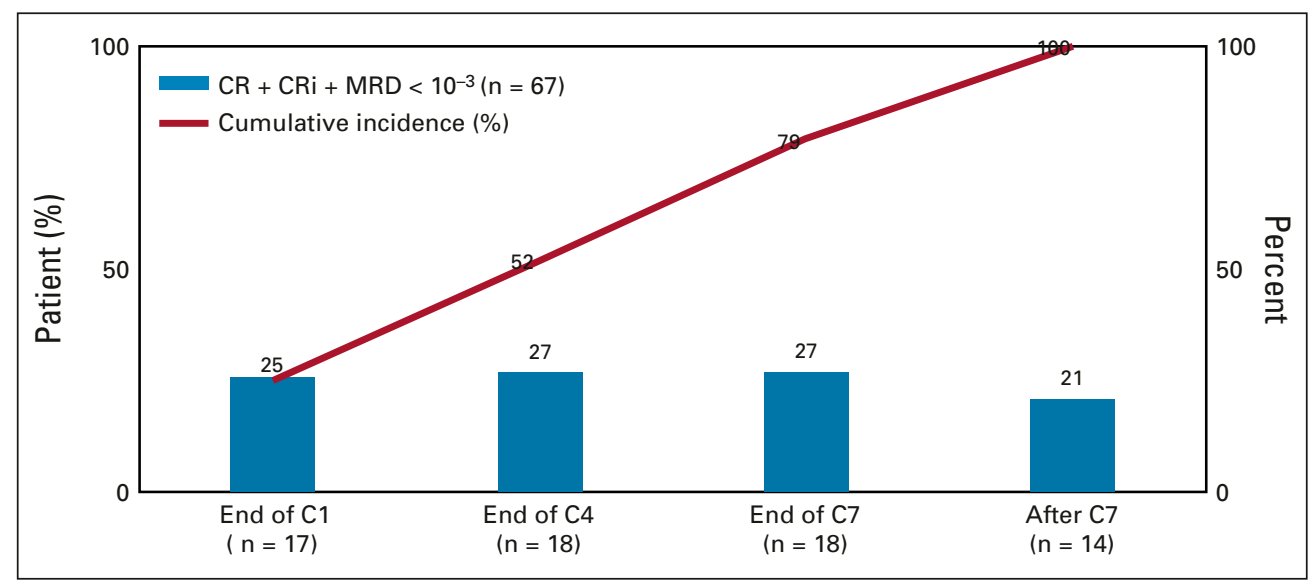

FIG 2. MRD by treatment cycle and cumulative incidence. End of $\mathrm{Cl}$ : $\mathrm{MRD}<10^{-3}$ from $\mathrm{C} 1$ day 1 to the end day of $\mathrm{C} 1+7$ days. End of $\mathrm{C} 4$ : MRD $<10^{-3}$ from the end day of $\mathrm{C} 1+8$ days to minimum value between end day of $\mathrm{C} 4$ and the last dose +7 days. End of $\mathrm{C} 7: \mathrm{MRD}<10^{-3}$ from the end day of $\mathrm{C} 4+1$ day to minimum value between end day of $\mathrm{C} 7$ and last dose +7 days. After $\mathrm{C} 7$ : end day of $\mathrm{C} 7+1$ day and onward up to cutoff date. $\mathrm{CR}$ was defined as the absolute neutrophil count $>10^{3} / \mu \mathrm{L}$, platelets $>10^{5} / \mu \mathrm{L}$, red cell transfusion independence, and bone marrow with $<5 \%$ blasts; $\mathrm{CRi}$ was defined as all criteria for $\mathrm{CR}$, except for neutropenia $\leq 10^{3} / \mu \mathrm{L}$ or thrombocytopenia $\leq 105 / \mu \mathrm{L}$. C, cycle; CR, complete remission; CRi, complete remission with incomplete hematologic remission; MRD, measurable residual disease. 


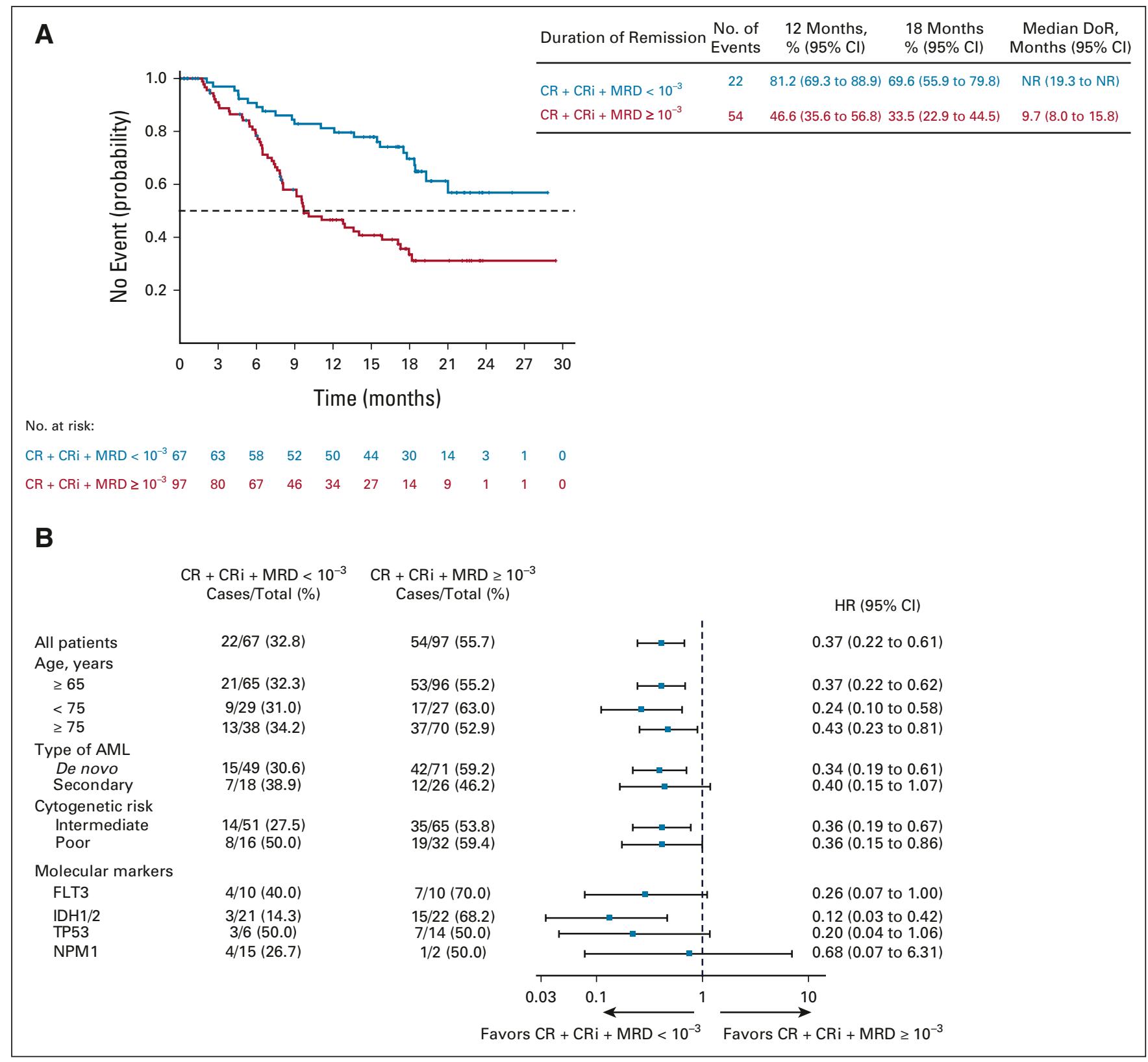

FIG 3. (A) DoR among patients with composite complete remission. (B) Forest plot for DoR in subgroups. CR, complete remissions; CRi, complete remission with incomplete hematologic recovery; DoR, duration of remission; MRD, measurable residual disease; NR, not reached.

median DoR for CRc was longer among patients who achieved an MRD-negative response than in those who had $M R D \geq 10^{-3}$ in all categories (Fig 3B and Data Supplement).

The median EFS for CRc was not reached in patients with an MRD-negative response, and the estimated 12-month EFS was $83.2 \%$ ( $95 \% \mathrm{Cl}, 71.6$ to 90.3). The median EFS was 10.6 months ( $95 \% \mathrm{Cl}, 9.0$ to 13.9 ) in patients with CRc and $\mathrm{MRD} \geq 10^{-3}$ (Figs $4 \mathrm{~A}$ and $4 \mathrm{~B}$ and Data Supplement). EFS was similar among patients who achieved $\mathrm{CRc}$ with an MRD-negative response after cycle 1 and thereafter (Fig 4C).

The median OS was not reached in patients with $\mathrm{CRc}$ and MRD-negative response, and the estimated 12-month OS was $94.0 \%$ (95\% Cl, 84.7 to 97.7$)$. The median OS was $18.7(95 \% \mathrm{Cl}, 12.9$ to not reached) months in patients with $\mathrm{CRC}$ and $\mathrm{MRD} \geq 10^{-3}$ (Figs $5 \mathrm{~A}$ and $5 \mathrm{~B}$ and Data Supplement). The results of the multivariate Cox regression analysis demonstrated a significant reduction in death rate with the achievement of $\mathrm{CRc}$ with MRD-negative response $(\mathrm{HR}=0.285 ; 95 \% \mathrm{Cl}, 0.159$ to $0.510 ; P<.001)$. Both $\mathrm{CR}$ and CRi with an MRD-negative response were significantly prognostic of OS (Fig 5C). Additional analysis showed that patients who achieved CRc with an MRD-negative response after cycle 1, and thereafter had similar baseline characteristics (Data Supplement), had significantly better 


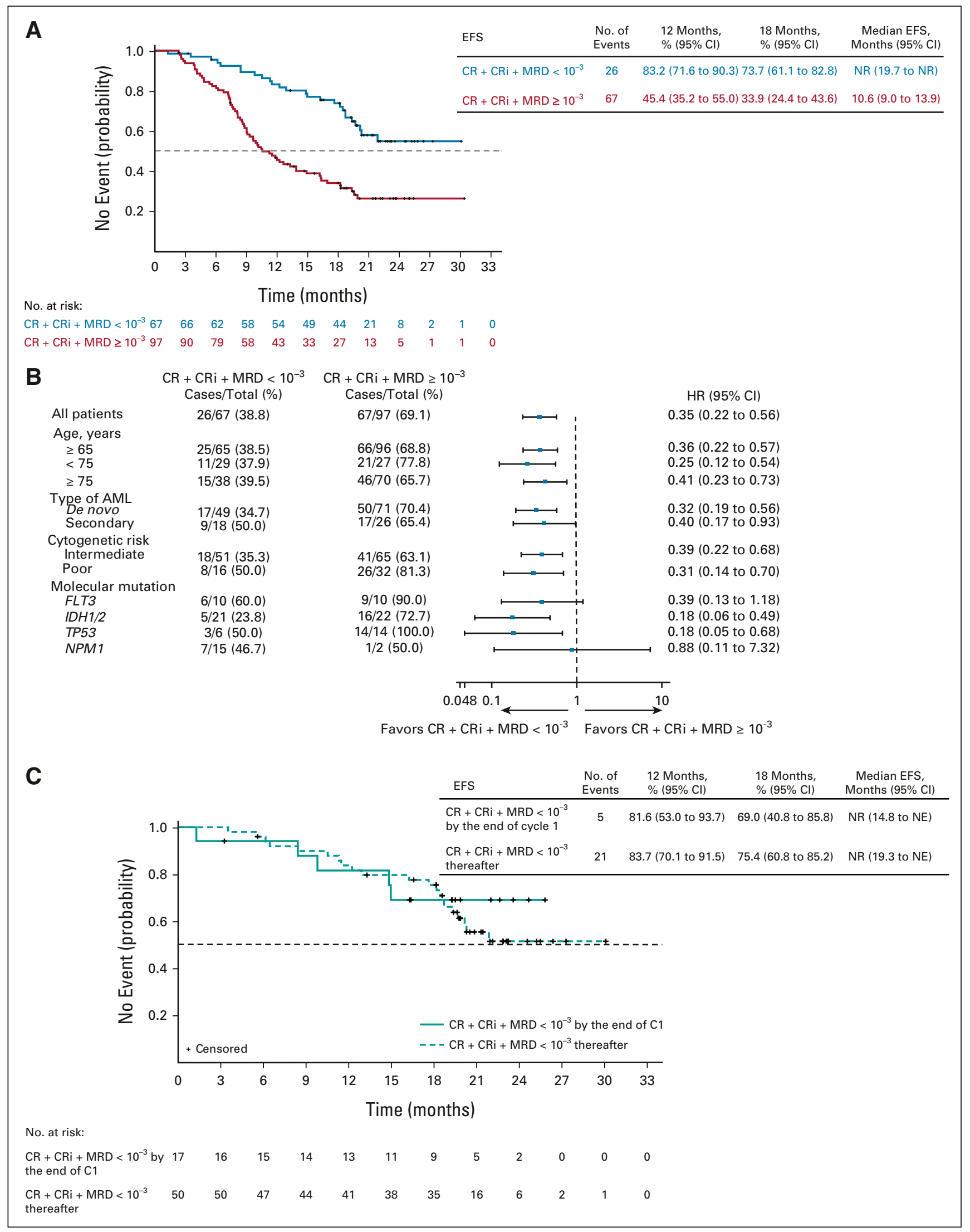

FIG 4. (A) EFS among patients. (B) Forest plot for EFS in subgroups. (C) Kaplan-Meier curves of EFS for patients who achieved composite complete remission and MRD-negative response by treatment cycles. $\mathrm{CR}$, complete remission; $\mathrm{CRi}$, complete remission with incomplete hematologic recovery; EFS, event-free survival; MRD, measurable residual disease; NE, not evaluable; NR, not reached. 
OS outcomes; the median OS was not reached in either group, and the estimated 12-month OS was $87.8 \%$ (95\% $\mathrm{Cl}, 59.5$ to 96.8) among patients who achieved an MRDnegative response after cycle 1 and $96.0 \%(95 \% \mathrm{Cl}, 84.9$ to 99.0) among patients who achieved an MRD-negative response thereafter (Fig 5D).

The common treatment-emergent $\geq$ grade 3 adverse events occurring in $\geq 15 \%$ of patients in either group were febrile neutropenia, neutropenia, thrombocytopenia, anemia, pneumonia, and leukopenia (Data Supplement). Numerically higher rates of grade $\geq 3$ neutropenia ( $54 \% \mathrm{v}$ $37 \%$ ) and febrile neutropenia ( $54 \%$ v $41 \%$ ) were noted in patients with $\mathrm{CRc}$ with an MRD-negative response versus patients with $\mathrm{CRC}$ and $\mathrm{MRD} \geq 10^{-3}$.

\section{DISCUSSION}

In the phase III VIALE-A trial, the combination treatment regimen of venetoclax and azacitidine resulted in superior remission rates and OS among treatment-naïve patients ineligible for intensive therapy. ${ }^{2}$ Among 190 patients who achieved $\mathrm{CRc}, 67$ also achieved an MRD $<10^{-3}$ response. The results of this exploratory analysis further demonstrated that patients who had achieved CRc with MRD-negative response had longer DoR for $\mathrm{CRC}$, EFS, and OS, than did patients who had MRD $\geq 10^{-3}$ in remission. This provides evidence that the lower-intensity combination of venetoclax and azacitidine can produce deep responses in patients ineligible for intensive therapy.

The achievement of an MRD-negative response predicted OS, even among patients with secondary AML. The median OS was not reached in either intermediate or poor cytogenetic risk groups attaining MRD-negative response, and the 12 -month estimated OS was $94 \%$ in both subgroups, indicating that obtaining an MRD-negative response, even in patients with adverse risk cytogenetics, confers durable patient outcomes. Consistent with this, in a recent study, Maiti et $\mathrm{al}^{16}$ evaluated the prognostic impact of baseline patient factors for response to another lower-intensity treatment regimen, venetoclax plus decitabine, and reported that both patients with intermediate- and poor-risk cytogenetics who achieved MRD-negative status enjoyed a superior OS. Taken together, these data suggest that initial post-treatment MRD-negative response may be a valuable prognostic marker of OS in patients who are treated with lower-intensity regimens.

Given that a quarter of patients on treatment achieved MRD responses by the end of cycle 1 , frequent late MRD responses were also observed; totally, $27 \%$ achieved an MRD-negative response by the end of cycle 7 , with a further $21 \%$ achieving a response thereafter. Thus, the timing of MRD response may be independent of the time of achievement of clinical remission and may occur well after the patient has achieved clinical remission.
Another study reported a strong prognostic correlation used in combination between MRD response and OS with venetoclax and decitabine and noted that the benefit of MRD-negative status was similar across 1-, 2-, and 4month time points. ${ }^{16}$ Furthermore, the median OS among patients who achieved an early versus late MRDnegative response was similar. Collectively, these results indicate that the attainment of a late MRD-negative response is not only possible but also associated with a better outcome. Consistent with this, unpublished data from the phase II studies before VIALE-A, in which MRD assessments were collected more frequently, indicate that MRD rates improved with longer time on therapy. Therefore, the first MRD values may not be indicative of the full effect of the venetoclax and azacitidine therapy. In addition, the current data show that patients who achieve later MRD negativity also have a survival advantage. For future studies, we would recommend at least two MRD assessments after achievement of remission.

We noted that the rates of neutropenia and febrile neutropenia were numerically higher in patients who achieved an MRD-negative response that warrants further investigation. However, these rates were similar to the overall study population of VIALE-A. ${ }^{2}$ Given the prognostic impact of an MRD-negative response on survival, with the proper use of prophylaxis and protocol-indicated cycle delays and dose reductions, the toxicities can be managed and ensure the safety of the regimen.

Molecular and MFC analyses are widely used techniques for MRD assessment in AML. ${ }^{11,13,17}$ The VIALE-A study is the first prospective study to show the utility of MRD assessment by MFC among patients treated with lowerintensity chemotherapy. Most patients were evaluable for MRD using MFC. In the venetoclax arm, of the 196 patients who achieved CRc, only 26 (13\%) had either missing or indeterminate MRD assessments, whereas in the placebo and azacitidine arm, 17\% (7 of 41) had missing MRD values.

In patients who present at diagnosis with genetic abnormalities associated with AML, the use of a combination of NGS and MFC has found that each technique had independent and additive prognostic value for predicting the rate of relapse and survival in younger (18-65 years) treatment-naive patients treated with intensive regimens. ${ }^{17}$ Future studies can explore the use of both NGS and MFC in treatment-naïve patients with AML treated with lowerintensity therapies.

The interpretation of the data presented here is subject to a few limitations. The small number of patients who achieved CRc with MRD-negative response in the placebo and azacitidine arms made it difficult to compare between intervention and placebo arms and warrants further research. In this study, MRD was collected at specified time points and not continuously monitored; hence, we could not 


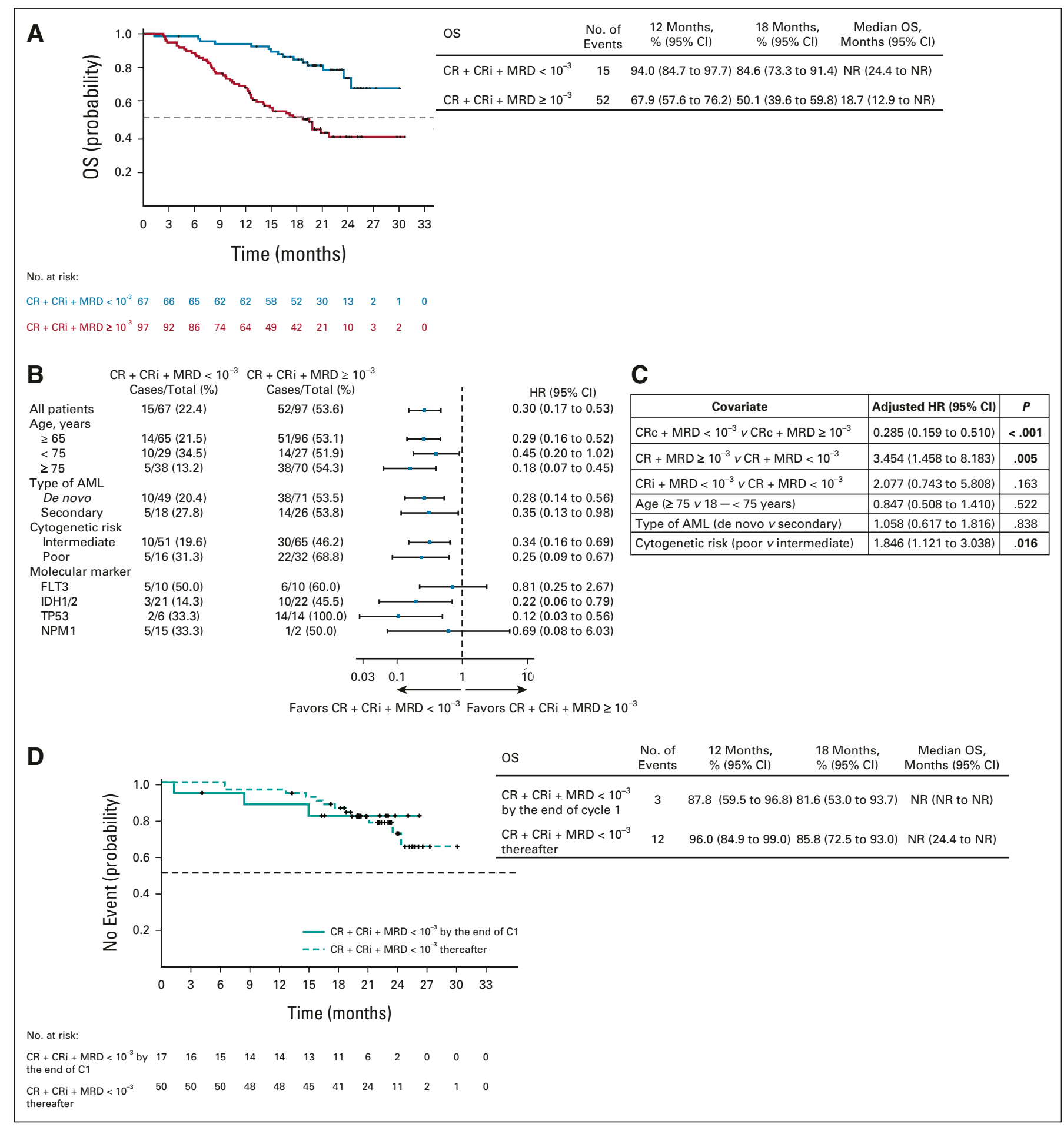

FIG 5. (A) Kaplan-Meier curves of OS among patients. (B) Forest plot for OS in subgroups. (C) Multivariate Cox regression analysis for OS. (D) Kaplan-Meier curves of OS for patients who achieved CRC and MRD-negative response by treatment cycles. AML, acute myeloid leukemia; C, cycle; CR, complete remission; CRc, composite complete remission; CRi, complete remission with incomplete hematologic recovery; HR, hazard ratio; MRD, measurable residual disease; NR, not reached; OS, overall survival.

establish the most informative time point for MRD assessment in this population. In addition, patients who had two successive disease assessments that indicated CR or $\mathrm{CRi}, \mathrm{BM}$ aspirates were no longer required for response or biomarker assessments; hence, it is possible that among these patients, MRD-negative responses may occur over time that warrants further evaluation. Furthermore, outcomes of the small numbers of patients in the molecular 
subsets should be interpreted with caution and warrant further prospective investigation.

For patients with AML ineligible for intensive therapy, the combination of venetoclax and azacitidine is now an established standard of care. Our observations reported herein establish the role of MRD assessment as an important predictor of outcomes in lower-intensity AML therapy and present an avenue for future investigation into patientspecific therapy on the basis of MRD response. Future studies of therapy deintensification/discontinuation in the MRD-negative subset may solidify the clinical utility of MRD

\section{AFFILIATIONS}

${ }^{1}$ Abramson Cancer Center, University of Pennsylvania, Philadelphia, PA ${ }^{2}$ Department of Internal Medicine, Division of Hematology and Oncology, University of California Davis School of Medicine, Sacramento, CA ${ }^{3}$ Department of Hematology and Hematopoietic Cell Transplantation and Gehr Family Center for Leukemia Research, City of Hope Comprehensive Cancer Center, Duarte, CA

${ }^{4}$ Centre Hospitalier Universitaire de Toulouse, Institut Universitaire du Cancer de Toulouse Oncopole, Université de Toulouse 3 Paul Sabatier, Toulouse, France

${ }^{5}$ Department of Medical Oncology and Hematology, Princess Margaret Cancer Centre, Toronto, Ontario, Canada

${ }^{6}$ Section of Hematology/Oncology, Department of Medicine, The University of Chicago Medicine, Chicago, IL

${ }^{7}$ Department of Medical Oncology, Dana-Farber Cancer Institute, Harvard Medical School, Boston, MA

${ }^{8}$ Department of Leukemia, Division of Cancer Medicine, The University of Texas MD Anderson Cancer Center, Houston, TX

${ }^{9}$ Department of Hematology, S. P. Botkin City Clinical Hospital, Moscow, Russia

${ }^{10}$ UOC Ematologia, Fondazione IRCCS Ca' Granda-Ospedale Maggiore Policlinico, Milan, Italy

${ }^{11}$ Department of Internal Medicine, China Medical University Hospital, Taichung, Taiwan

${ }^{12}$ Department of Hematology-Oncology, Samsung Medical Center, Sungkyunkwan University School of Medicine, Seoul, Korea

${ }^{13}$ Department of Hematology, Ankara University School of Medicine, Ankara, Turkey

${ }^{14}$ Department of Hematology and Cell Therapy, Aichi Cancer Center Hospital, Nagoya, Japan

${ }^{15}$ University of Debrecen, Faculty of Medicine, Department of Hematology, Debrecen, Hungary

${ }^{16}$ AbbVie Inc., North Chicago, IL

${ }^{17}$ Genentech Inc., South San Francisco, CA

${ }^{18}$ Department of Internal Medicine III, UIm University Hospital, UIm, Germany

\section{CORRESPONDING AUTHOR}

Keith W. Pratz, MD, Abramson Cancer Center, Perelman Center for Advanced Medicine, West Pavilion, 4th Floor, 3400 Civic Center Boulevard, Philadelphia, PA 19104; e-mail: keith.pratz@pennmedicine. upenn.edu.

\section{CLINICAL TRIAL INFORMATION} NCT02993523 monitoring in the unfit-for-intensive-chemotherapy subset, with hopes of identifying a subset with long-term EFS. Similarly, in the MRD-positive subset, future studies of the addition of novel agents to this regimen, with the end point of achieving MRD conversion, would be of high interest, as the bulk of patients treated with azacitidine and venetoclax remain MRD-positive despite the achievement of CRc. This study demonstrated the importance and utility of MRD monitoring as a prognostic factor for OS in the setting of lower-intensity therapy and warrants further investigation to establish its role in the management of patients with AML.

\section{AUTHORS' DISCLOSURES OF POTENTIAL CONFLICTS OF INTEREST}

Disclosures provided by the authors are available with this article at DOI https://doi.org/10.1200/JC0.21.01546.

\section{DATA SHARING STATEMENT}

These clinical trial data can be requested by any qualified researchers who engage in rigorous, independent scientific research and will be provided following review and approval of a research proposal and Statistical Analysis Plan (SAP) and execution of a Data Sharing Agreement (DSA). Data requests can be submitted at any time, and the data will be accessible for 12 months, with possible extensions considered. For more information on the process, or to submit a request, visit the following link: https://www.abbvie.com/our-science/clinicaltrials/clinical-trials-data-and-information-sharing/data-and-informationsharing-with-qualified-researchers.html.

\section{AUTHOR CONTRIBUTIONS}

Conception and design: Keith W. Pratz, Brian A. Jonas, Michael J. Thirman, Jun Ho Jang, Monique Dail, Brenda Chyla, Jalaja Potluri, Hartmut Döhner

Provision of study materials or patients: Vinod Pullarkat, Christian Recher, Jacqueline S. Garcia, Courtney D. DiNardo, Vladimir Vorobyev, Su-Peng Yeh, Kazuhito Yamamoto, Arpad Illes, Hartmut Döhner

Collection and assembly of data: Keith W. Pratz, Brian A. Jonas, Christian Recher, Andre C. Schuh, Michael J. Thirman, Jacqueline S. Garcia, Courtney D. DiNardo, Vladimir Vorobyev, Nicola S. Fracchiolla, Su-Peng Yeh, Jun Ho Jang, Muhit Ozcan, Kazuhito Yamamoto, Arpad Illes, Brenda Chyla, Jalaja Potluri

Data analysis and interpretation: Keith W. Pratz, Brian A. Jonas, Vinod Pullarkat, Christian Recher, Andre C. Schuh, Michael J. Thirman, Jacqueline S. Garcia, Jun Ho Jang, Muhit Ozcan, Kazuhito Yamamoto, Arpad Illes, Ying Zhou, Monique Dail, Brenda Chyla, Jalaja Potluri, Hartmut Döhner

Manuscript writing: All authors

Final approval of manuscript: All authors

Accountable for all aspects of the work: All authors

\section{ACKNOWLEDGMENT}

The authors would like to thank the patients and their families, the study coordinators, and the support staff. The authors would also like to acknowledge all investigators of Study M15-656. Medical writing support was provided by Dalia Majumdar, PhD, an employee of AbbVie. 


\section{REFERENCES}

1. National Comprehensive Cancer Network: NCCN Guidelines for Patients with Acute Myeloid Leukemia, Version 3.2021, 2021. https://www.nccn.org/ professionals/physician_gls/pdf/aml.pdf

2. DiNardo CD, Jonas BA, Pullarkat V, et al: Azacitidine and venetoclax in previously untreated acute myeloid leukemia. N Engl J Med 383:617-629, 2020

3. Appelbaum FR, Gundacker H, Head DR, et al: Age and acute myeloid leukemia. Blood 107:3481-3485, 2006

4. Kantarjian H, O'Brien S, Cortes J, et al: Results of intensive chemotherapy in 998 patients age 65 years or older with acute myeloid leukemia or high-risk myelodysplastic syndrome: Predictive prognostic models for outcome. Cancer 106:1090-1098, 2006

5. Freeman SD, Jovanovic JV, Grimwade D: Development of minimal residual disease-directed therapy in acute myeloid leukemia. Semin Oncol 35:388-400, 2008

6. Buccisano F, Maurillo L, Piciocchi A, et al: Minimal residual disease negativity in elderly patients with acute myeloid leukemia may indicate different postremission strategies than in younger patients. Ann Hematol 94:1319-1326, 2015

7. Terwijn M, van Putten WL, Kelder A, et al: High prognostic impact of flow cytometric minimal residual disease detection in acute myeloid leukemia: Data from the HOVON/SAKK AML 42A study. J Clin Oncol 31:3889-3897, 2013

8. Freeman SD, Virgo P, Couzens S, et al: Prognostic relevance of treatment response measured by flow cytometric residual disease detection in older patients with acute myeloid leukemia. J Clin Oncol 31:4123-4131, 2013

9. Boddu P, Jorgensen J, Kantarjian H, et al: Achievement of a negative minimal residual disease state after hypomethylating agent therapy in older patients with AML reduces the risk of relapse. Leukemia 32:241-244, 2018

10. Döhner H, Estey E, Grimwade D, et al: Diagnosis and management of AML in adults: 2017 ELN recommendations from an international expert panel. Blood 129:424-447, 2017

11. Schuurhuis GJ, Heuser M, Freeman S, et al: Minimal/measurable residual disease in AML: A consensus document from the European LeukemiaNet MRD Working Party. Blood 131:1275-1291, 2018

12. Thermo Fisher Scientific: Real-Time PCR. https://www.thermofisher.com/us/en/home/life-science/pcr/real-time-pcr.html?cid=corp_ask-a-scientist_blog

13. Dix C, Lo TH, Clark G, et al: Measurable residual disease in acute myeloid leukemia using flow cytometry: A review of where we are and where we are going. J Clin Med 9:1714, 2020

14. Cheson BD, Bennett JM, Kopecky KJ, et al: Revised recommendations of the International Working Group for diagnosis, standardization of response criteria, treatment outcomes, and reporting standards for therapeutic trials in acute myeloid leukemia. J Clin Oncol 21:4642-4649, 2003

15. US Department of Health and Human Services: National Cancer Institute: Common Terminology Criteria for Adverse Events (CTCAE) v4.03, 2018. https:// ctep.cancer.gov/protocolDevelopment/electronic_applications/ctc.htm

16. Maiti A, DiNardo CD, Wang SA, et al: Prognostic value of measurable residual disease after venetoclax and decitabine in acute myeloid leukemia. Blood Adv 5: 1876-1883, 2021

17. Jongen-Lavrencic M, Grob T, Hanekamp D, et al: Molecular minimal residual disease in acute myeloid leukemia. N Engl J Med 378:1189-1199, 2018 


\section{Measurable Residual Disease Response and Prognosis in Treatment-Naïve Acute Myeloid Leukemia With Venetoclax and Azacitidine}

The following represents disclosure information provided by authors of this manuscript. All relationships are considered compensated unless otherwise noted. Relationships are self-held unless noted. I = Immediate Family Member, Inst = My Institution. Relationships may not relate to the subject matter of this manuscript. For more information about ASCO's conflict of interest policy, please refer to www.asco.org/rwc or ascopubs.org/jco/authors/author-center.

Open Payments is a public database containing information reported by companies about payments made to US-licensed physicians (Open Payments).

\section{Keith W. Pratz}

Consulting or Advisory Role: Astellas Pharma, Boston Biomedical, AbbVie Research Funding: Agios (Inst), Millennium (Inst), AbbVie (Inst), Daiichi Sankyo (Inst)

\section{Brian A. Jonas}

Consulting or Advisory Role: AbbVie, Jazz Pharmaceuticals, GlycoMimetics, Treadwell Therapeutics, Takeda, Genentech, Bristol Myers Squibb/Pfizer, Pfizer Research Funding: Daiichi Sankyo (Inst), AbbVie (Inst), Pharmacyclics (Inst), Genentech/Roche (Inst), Glycomimetics (Inst), Celgene (Inst), FORMA Therapeutics (Inst), Incyte (Inst), Arog (Inst), Roche (Inst), Jazz Pharmaceuticals (Inst), Pfizer (Inst), Sigma-Tau (Inst), Forty Seven, Amgen (Inst), Immune-Onc Therapeutics (Inst), Loxo (Inst), Gilead/Forty Seven (Inst)

Travel, Accommodations, Expenses: AbbVie

\section{Vinod Pullarkat}

Consulting or Advisory Role: Novartis, Amgen, Pfizer, Jazz Pharmaceuticals, AbbVie/Genentech

Speakers' Bureau: Novartis, Amgen, Jazz Pharmaceuticals, AbbVie

\section{Christian Recher}

Consulting or Advisory Role: Celgene, Amgen, Novartis, Jazz Pharmaceuticals, AbbVie, Janssen, Astellas Pharma, MacroGenics, Daiichi Sankyo, Otsuka, Novartis, Incyte, Pfizer, Roche, Takeda

Research Funding: Celgene (Inst), Amgen (Inst), Jazz Pharmaceuticals (Inst), Astellas Pharma (Inst), Agios (Inst), Daiichi Sankyo (Inst), MaaT Pharma (Inst), Roche (Inst), AbbVie (Inst), Novartis (Inst), Chugai Pharma (Inst), IQVIA (Inst) Travel, Accommodations, Expenses: Incyte, Celgene, Sanofi, Amgen, Novartis, Daiichi Sankyo, Gilead Sciences

Andre C. Schuh

Honoraria: Celgene, Amgen, Pfizer, Novartis, Jazz Pharmaceuticals

Research Funding: Celgene, Amgen, Agios, Pfizer

Michael J. Thirman

Consulting or Advisory Role: AstraZeneca, Genentech, AbbVie, Adaptive Biotechnologies, Celgene Pharmacyclics

Research Funding: AbbVie (Inst), Syndax (Inst), Merck (Inst), TG Therapeutics (Inst)

Jacqueline S. Garcia

Consulting or Advisory Role: AbbVie, Takeda, Astellas Pharma

Research Funding: AbbVie (Inst), Pfizer (Inst), Genentech/Roche (Inst), Lilly (Inst), AstraZeneca (Inst), Prelude Therapeutics (Inst)

Courtney D. DiNardo

This author is a member of the Journal of Clinical Oncology Editorial Board Journal policy recused the author from having any role in the peer review of this manuscript.

Leadership: Notable Labs

Stock and Other Ownership Interests: Notable Labs

Honoraria: Agios, Celgene, AbbVie, Jazz Pharmaceuticals, Daiichi Sankyo, Novartis, Takeda

Consulting or Advisory Role: Celgene, Agios, AbbVie

Research Funding: AbbVie, Agios, Celgene, Daiichi Sankyo

Vladimir Vorobyev

Consulting or Advisory Role: AstraZeneca, Janssen Oncology, Sanofi, Novartis, BeiGene, Roche, AbbVie, Takeda, Gilead Sciences

Speakers' Bureau: AbbVie, AstraZeneca, Amgen, BioCad, Janssen, Sanofi,

Takeda Novartis, BeiGene

Travel, Accommodations, Expenses: AstraZeneca
Nicola S. Fracchiolla

Consulting or Advisory Role: Amgen, Jazz Pharmaceuticals, Incyte, AbbVie Travel, Accommodations, Expenses: Pfizer

\section{Su-Peng Yeh}

Honoraria: Novartis, Bristol Myers Squibb, Janssen, Takeda, AbbVie, Amgen, Sanofi, Bayer, Astellas Pharma

Consulting or Advisory Role: AbbVie, Sanofi, Novartis, Pfizer, Chugai Pharma

Jun Ho Jang

Speakers' Bureau: Novartis

Muhit Ozcan

Research Funding: Janssen, Celgene, Takeda, Bayer, Merck, Archigen Biotech, Roche, AbbVie, Acerta Pharma/AstraZeneca

\section{Kazuhito Yamamoto}

Honoraria: Kyowa Hakko Kirin, Takeda, Janssen, Bristol Myers Squibb, Celgene, Sumitomo Dainippon, Ono Pharmaceutical, Chugai Pharma, Novartis, Otsuka, Mundipharma, Eisai, MSD, Meiji Seika Kaisha, Sanofi, Nippon Shinyaku, AbbVie, GlaxoSmithKline, SymBio Pharmaceuticals, Micron, Daiichi Sankyo Consulting or Advisory Role: Ono Pharmaceutical, Meiji Seika Kaisha, Chugai Pharma, Bristol Myers Squibb, Kyowa Hakko Kirin, Takeda, Celgene, HUYA Bioscience International, Stemline Therapeutics, Eisai, Janssen, AstraZeneca Daiichi Sankyo, AbbVie,

Research Funding: Chugai Pharma (Inst), Novartis (Inst), ARIAD (Inst), Takeda (Inst), Gilead Sciences (Inst), AbbVie (Inst), Ono Pharmaceutical (Inst), Celgene (Inst), Solasia Pharma (Inst), MSD (Inst), Eisai (Inst), Zenyaku Kogyo (Inst), Bayer (Inst), SymBio Pharmaceuticals (Inst), AstraZeneca (Inst), Incyte (Inst), Mundipharma (Inst), Yakult Pharmaceutical (Inst)

\section{Arpad Illes}

Consulting or Advisory Role: Janssen, Takeda, Novartis, Pfizer, Roche, Celgene Research Funding: Takeda, Seattle Genetics

Travel, Accommodations, Expenses: Novartis, Janssen, Pfizer, Roche

Ying Zhou

Stock and Other Ownership Interests: AbbVie

Monique Dail

Employment: Genentech/Roche

Stock and Other Ownership Interests: Roche/Genentech

Brenda Chyla

Employment: AbbVie

Stock and Other Ownership Interests: AbbVie

Jalaja Potluri

Employment: AbbVie

Stock and Other Ownership Interests: AbbVie

Hartmut Döhner

Consulting or Advisory Role: AbbVie, Agios, Amgen, Astellas Pharma, Celgene, Jazz Pharmaceuticals, Janssen Oncology, Novartis, AstraZeneca, BerlinChemie, GEMoaB, Bristol Myers Squibb/Celgene, Gilead Sciences, Syndax Research Funding: Arog, Amgen, Bristol Myers Squibb, Novartis, Pfizer, Jazz Pharmaceuticals, AbbVie, Kronos Bio

No other potential conflicts of interest were reported. 